\title{
Signatures of Gravitational Fixed Points at the Large Hadron Collider
}

\author{
Daniel F. Litim ${ }^{1,2}$ and Tilman Plehn ${ }^{3}$ \\ ${ }^{1}$ Department of Physics and Astronomy, University of Sussex, Brighton, BN1 9QH, United Kingdom \\ ${ }^{2}$ Theory Group, Physics Division, CERN, CH-1211 Geneva 23, Switzerland \\ ${ }^{3}$ SUPA, School of Physics, University of Edinburgh, United Kingdom
}

(Received 31 July 2007; published 1 April 2008)

\begin{abstract}
We study quantum-gravitational signatures at the CERN Large Hadron Collider (LHC) in the context of theories with extra spatial dimensions and a low fundamental Planck scale in the TeV range. Implications of a gravitational fixed point at high energies are worked out using Wilson's renormalization group. We find that relevant cross sections involving virtual gravitons become finite. Based on gravitational lepton pair production we conclude that the LHC is sensitive to a fundamental Planck scale of up to $6 \mathrm{TeV}$.
\end{abstract}

DOI: 10.1103/PhysRevLett.100.131301

Introduction. - The fascinating idea that the fundamental scale of gravity, the Planck scale, is connected to the scale of electroweak symmetry breaking-within a higher-dimensional setting-has stimulated extensive model building and phenomenology [1]. Central to these scenarios is that gravity propagates in the higherdimensional bulk, while standard model (SM) particles are often confined to the four-dimensional brane. Most interestingly, the hypothesis of a fundamental Planck scale in the TeV energy range can be tested at high-energy colliders. In this way, the LHC becomes sensitive to the dynamics of gravity, and could possibly become the first experiment able to establish evidence for the quantization of gravity.

The phenomenology of quantum gravity at hadron colliders [2-4] is based on direct graviton production like $p p \rightarrow$ jet $+G$, leading to missing energy signatures, virtual graviton exchange in standard model reference processes like $p p \rightarrow \ell^{+} \ell^{-}$, leading to deviations in critical distributions, and the production and decay of mini-black holes. The first two processes are standard search channels for physics beyond the SM. In the context of low-scale quantum gravity, they have been studied within effective field theory [2], which allows for a well-controlled description as long as the relevant momentum scales are sufficiently below an ultraviolet (UV) cutoff of the order of the fundamental Planck scale $M_{D}$. Near the Planck scale and above, an understanding of gravitational interactions requires an explicit quantum theory for gravity. It has been suggested that a local quantum theory of gravity in terms of the metric field may very well exist on a nonperturbative level [5], despite its perturbative nonrenormalizability. This asymptotic safety scenario requires the existence of a nontrivial UV fixed point for quantum gravity under the renormalization group (RG). In four dimensions, growing evidence for a UV fixed point has arisen recently, based on RG studies [6-10] (see [11] for reviews) and lattice simulations [12]. This picture has also been extended to higher dimensions $[10,13]$. Hence, asymptotically safe gravity
PACS numbers: 04.60. $-\mathrm{m}, 04.50 .-\mathrm{h}, 11.10 . \mathrm{Hi}, 11.15 . \mathrm{Tk}$ provides an excellent starting point to access the quantum-gravitational domain.

In this Letter, we study implications of gravitational fixed points on lepton pair production $p p \rightarrow \ell^{+} \ell^{-}$through graviton exchange in scenarios with large extra dimensions. The main new ingredient is the nontrivial RG running of the gravitational sector in higher dimensions $[10,13]$. We first discuss basic implications of a gravitational fixed point. We then show that fixed point scaling leads to a finite virtual graviton amplitude. We compute the effective cross sections and the $5 \sigma$ discovery reach for the fundamental Planck scale at LHC energies.

Gravitational fixed point. - We begin with a discussion of structural implications of gravitational fixed points and consider a Callan-Symanzik type equation for the gravitational coupling $G$ with respect to a momentum scale $\mu$ in $D$ dimensions $[10,11]$. In terms of the running dimensionless gravitational coupling $g(\mu)=G(\mu) \mu^{D-2} \equiv$ $G_{0} Z_{G}^{-1}(\mu) \mu^{D-2}$, it reads

$$
\beta_{g} \equiv \frac{d g(\mu)}{d \ln \mu}=(D-2+\eta) g(\mu)
$$

Here $\eta=-\mu \partial_{\mu} \ln Z_{G}$ denotes the anomalous dimension of the graviton. We assume that the fundamental action is local in the metric field. The wave function factor is normalized as $Z_{G}\left(\mu_{0}\right)=1$ at some reference scale $\mu_{0}$ with $G\left(\mu_{0}\right)$ given by Newton's constant $G_{0}$. In general, the anomalous dimension depends on all couplings of the theory. Because of its structure, (1) can achieve two types of fixed points. At small coupling, the anomalous dimension vanishes and $g=0$ corresponds to the noninteracting (i.e., Gaussian) fixed point of (1). This fixed point dominates the deep infrared (IR) region of gravity where $\mu \rightarrow$ 0 . In turn, an interacting fixed point $g_{*}$ may be achieved if the anomalous dimension of the graviton becomes nonperturbatively large,

$$
\eta_{*}=2-D
$$

Hence, a nontrivial fixed point of quantum gravity in 
$D>2$ implies a negative integer value for the graviton anomalous dimension, precisely counterbalancing the canonical dimension of $G$. This behavior implies that the gravitational coupling constant scales as $G(\mu) \rightarrow$ $g_{*} / \mu^{D-2}$ in the vicinity of a nontrivial fixed point. In the UV limit where $\mu \rightarrow \infty$, the gravitational coupling $G(\mu)$ becomes arbitrarily weak.

Wilson's renormalization group.-Now we turn to RG flows for gravity [6] and consider the effective action $\Gamma_{k}$

$$
\Gamma_{k}=\frac{1}{16 \pi G_{k}} \int d^{D} x \sqrt{g}[-R(g)+\cdots],
$$

where $k$ denotes the Wilsonian RG scale replacing the scale $\mu$ introduced in (1), and $R(g)$ denotes the Ricci scalar. The dots in (3) stand for the cosmological constant, higher-dimensional operators in the metric field, gravitymatter interactions, a classical gauge fixing, and ghost terms. In Wilson's approach, all couplings in (3) become running couplings as functions of the momentum scale $k$. For $k \ll M_{D}$, the gravitational sector is well approximated by the Einstein-Hilbert action with $G_{k} \approx G_{0}$, and similarly for the gravity-matter couplings. The corresponding operators scale canonically. At $k \approx M_{D}$ and above, the nontrivial RG running of gravitational couplings becomes important. The Wilsonian RG flow for an action (3) is given by an exact differential equation $[6,14]$

$$
\partial_{t} \Gamma_{k}=\frac{1}{2} \operatorname{Tr}\left(\Gamma_{k}^{(2)}+R_{k}\right)^{-1} \partial_{t} R_{k}
$$

and $t=\ln k$. The trace stands for a momentum integration and a sum over indices and fields, and $R_{k}\left(q^{2}\right)$ denotes an appropriate infrared cutoff function at momentum scale $q^{2} \approx k^{2}$ [15]. Diffeomorphism invariance under local coordinate transformations is controlled by modified Ward identities [6], similar to those known for non-Abelian gauge theories [16].

Running couplings. - To illustrate the main RG effects we approximate (3) by the Ricci scalar and discuss the running of $g_{k}$, following [10]. The inclusion of a cosmological constant modifies the approach towards fixed point scaling [13] without altering the central pattern relevant for the discussion below. Using (3) and (4), we find [10]

$$
\beta_{g}=\frac{(1-4 D g)(D-2) g}{1-(2 D-4) g}, \quad \eta=\frac{2(D-2)(D+2) g}{2(D-2) g-1},
$$

where $g$ has been rescaled by a numerical factor. We observe a Gaussian fixed point, and a non-Gaussian one at $g_{*}=1 /(4 D)$. Integrating the flow (5) gives

$$
\frac{1}{D-2} \ln \left|\frac{g_{k}}{g_{0}}\right|-\frac{1}{\theta_{\mathrm{NG}}} \ln \left|\frac{g_{*}-g_{k}}{g_{*}-g_{0}}\right|=\ln \frac{k}{k_{0}},
$$

with initial condition $g_{0}$ at $k=k_{0}$, and $\theta_{\mathrm{NG}}=2 D \frac{D-2}{D+2}$. The result (6) holds for generic momentum cutoff, only $g_{*}$ and the scaling exponent $\theta_{\mathrm{NG}}$ depend slightly on the cutoff choice $[10,13]$. Inserting the running coupling (6) into (5) shows that the anomalous dimension displays a smooth crossover between the IR domain $k \ll k_{\text {tr }}$ where $\eta \approx 0$ and the UV domain $k \gg k_{\text {tr }}$ where $\eta \approx 2-D$ (see Fig. 1), and $k$ appropriately normalized with $k_{\mathrm{tr}}$ of the order of the Planck scale. We note that the crossover regime becomes narrower with increasing dimension.

Gravitational dilepton production. -Now we turn to scenarios with large extra dimensions where gravity propagates in the $D$ dimensional bulk and matter fields live on a four-dimensional brane [1]. The four-dimensional Planck scale $M_{\mathrm{Pl}}$ is related to the size $L$ of the $n$ extra dimensions and the $D$-dimensional Planck scale $M_{D}$ as $M_{\mathrm{Pl}} \sim$ $M_{D}^{2}\left(M_{D} L\right)^{n}$. A fundamental Planck scale in the TeV range requires $1 / L \ll M_{D}$. Virtual graviton effects, as opposed to real graviton emission, are more sensitive to an underlying UV fixed point [2]. Hence we consider dilepton production through virtual gravitons. To lowest order in canonical dimension, this is generated through an effective dimension-8 operator in the effective action, involving four fermions and a graviton [2,17,18]. Tree-level graviton exchange is described by an amplitude $\mathcal{A}=\mathcal{S} \cdot \mathcal{T}$, where $\mathcal{T}=T_{\mu \nu} T^{\mu \nu}-\frac{1}{2+n} T_{\mu}^{\mu} T_{\nu}^{\nu}$ is a function of the energymomentum tensor, and

$$
\mathcal{S}=\frac{S_{n-1}}{M_{D}^{2+n}} \int_{0}^{\infty} d m m^{n-1} P(s, m)
$$

with $S_{n-1}=2 \pi^{n / 2} / \Gamma(n / 2)$ is a function of the scalar part $P(s, m)$ of the graviton propagator $[2,17,18]$. The integration over the Kaluza-Klein (KK) masses $m$, which we can take as continuous, reflects that gravity propagates in the higher-dimensional bulk. If the graviton anomalous dimension is small $|\eta| \ll 1$, the propagator is

$$
P(s, m)=\left(s+m^{2}\right)^{-1} .
$$

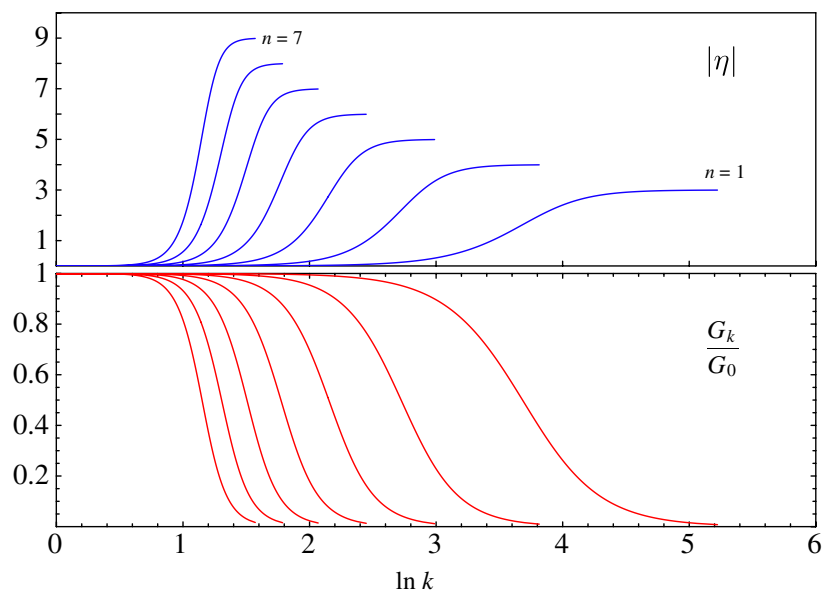

FIG. 1 (color online). Running anomalous dimensions (upper panel) and gravitational couplings (lower panel) from (5) and (6) for $D=4+n$ dimensions with $n=1, \ldots, 7$ (from right to left). The crossover takes place at the respective Planck scale. 

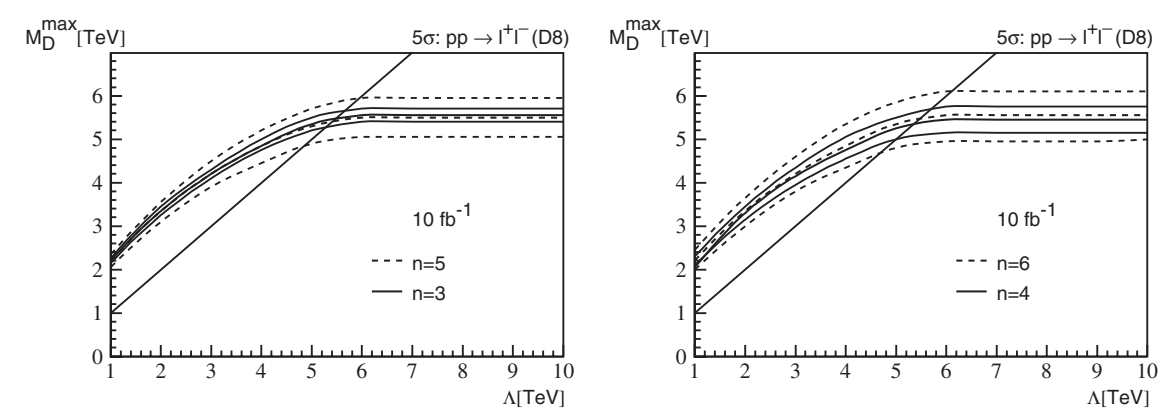

FIG. 2. The $5 \sigma$ discovery contours in $M_{D}$ at the LHC, shown as a function of a cutoff $\Lambda$ on $E_{\text {parton }}$ for an assumed integrated luminosity of $10 \mathrm{fb}^{-1}$. Thin lines show a $\pm 10 \%$ variation of $k_{\text {tr }}$ about $M_{D}$, the straight line is $M_{D}^{\max }=\Lambda$. The leveling off at $M_{D}^{\max } \approx \Lambda$ reflects the gravitational UV fixed point. To enhance the reach we require $m_{\ell \ell}^{\min }=$ $\min \left(M_{D} / 3,2 \mathrm{TeV}\right)$.
This is valid if the relevant momentum transfer is $\ll M_{D}$. It is well known that (7) with (8) is UV divergent for $n \geq 2$ [2]. Implementing an UV cutoff $\Lambda$ [17] as the upper integration boundary in (7), and using (8), gives

$$
\mathcal{S}_{\Lambda}=\frac{S_{n-1}}{n-2} \frac{1}{M_{D}^{4}}\left(\frac{\Lambda}{M_{D}}\right)^{n-2}\left[1+\mathcal{O}\left(\frac{s}{\Lambda^{2}}\right)\right] .
$$

The strong cutoff dependence shows that (7) with (8) is dominated in the UV by the Kaluza-Klein modes, if $n \geq 2$. In Wilson's approach (4), the graviton's anomalous dimension $\eta$ and its scale dependence have to be taken into account. Technically this is implemented by matching the RG scale $k^{2}$ with $p^{2}=s+m^{2}$, the relevant scale of the propagator entering (7), and $k_{\text {tr }}$ of the order of the Planck scale. For the dressed propagator $1 /\left(Z_{G}(k) p^{2}\right)$, this leads to a behavior $\sim p^{-2(1-\eta(p) / 2)}$. With $(2)$, this becomes $\sim\left(p^{2}\right)^{-D / 2}$ for large $p^{2}$, as opposed to the $p^{-2}$ behavior in the perturbative domain. Applying this RG improvement to $(8)$, we are lead to

$$
P(s, m)=\frac{k_{\mathrm{tr}}^{n+2}}{\left(s+m^{2}\right)^{n / 2+2}}
$$

in the vicinity of an UV fixed point. The observation central for our purposes is that (7) with (10) is finite even in the UV limit of the integration. The large anomalous dimensions in asymptotically safe gravity suffices to provide for a finite dilepton production rate.

We emphasize that our RG improvement is conceptually different from the form factor introduced in [19]. The latter employs the matching $k^{2}=s$, implying that Kaluza-Klein modes are treated perturbatively. Consequently, and unlike here, the form factor method [19] still requires an UV cutoff to render (7) finite, analogous to (9) within effective field theory.

Results. - The task to evaluate dilepton rates via the RG improvement of (7) is simplified by noticing that the anomalous dimension displays a rapid crossover from IR to UV scaling (see Fig. 1), in particular, in higher dimensions. Therefore we use (8) in the IR regime where $k<k_{\text {tr }}$ and (10) for the UV regime $k>k_{\mathrm{tr}}, k_{\mathrm{tr}}=M_{D}$. Since the integral at hadron colliders is sensitive to a gluon-density induced bias towards the smallest possible $s$, we retain for simplicity the leading term in $s / M_{D}^{2}$ in either regime.
In Fig. 2 we display the discovery potential in $M_{D}$ at the LHC. Taking into account the leading backgrounds we compute the minimal signal cross section for which a $5 \sigma$ excess is observed, assuming statistical errors. (See [18] for technical details.) This minimal cross section translates into a maximum reach $M_{D}^{\max }$. Consistency is checked by introducing an artificial cutoff $\Lambda$ on the partonic energy [18], setting the partonic signal cross section to zero for $E_{\text {parton }}>\Lambda$. This cutoff is not required in our approach and for sufficiently large $\Lambda, M_{D}^{\max }$ becomes independent of it. This is nicely seen in Fig. 2 once $\Lambda>M_{D}$. The fact that $M_{D}^{\max }$ levels off at about $\Lambda \approx M_{D}^{\max }$ reflects the onset of the underlying UV fixed point. To estimate uncertainties in our approximations, we allow for a $10 \%$ variation in $k_{\mathrm{tr}}$, leading to mild variations in Fig. 2 of a similar magnitude, slightly increasing with $n$.

In Fig. 3 we show the normalized $E_{\text {parton }}$ distributions for Drell-Yan production including KK gravitons for $n=3$ and $M_{D}=5$ and $8 \mathrm{TeV}$. In contrast to Fig. 2 we do not apply any $m_{\ell \ell}$ cut here. The solid curves represent our fixed point analysis. The entire $E_{\text {parton }}$ range contributes to the rate as long as there is a sizeable parton luminosity. The dashed curves correspond to the approximation (9) with UV cutoff $\Lambda=M_{D}$. Obviously, there are no contributions above $E_{\text {parton }}=\Lambda$. The two sets of curves do not scale in a simple manner because standard model and KK amplitudes

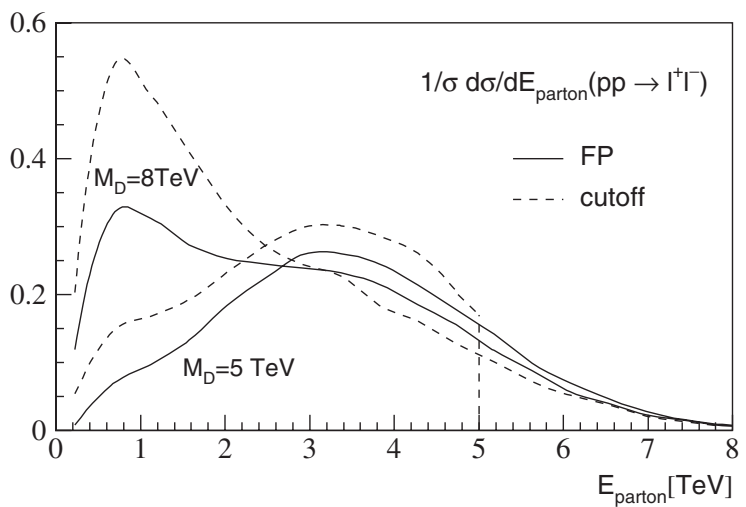

FIG. 3. Comparison of normalized distributions of the partonic energy $E_{\text {parton }}$ for the dimension- 8 operator correction to DrellYan production at the LHC $(n=3)$. Full line: present work, dashed line: approximation (9) with $\Lambda=M_{D}$. 
TABLE I. Comparison of dilepton production rates at the LHC after acceptance cuts. See main text for the definitions of (a), (b) and (c). All rates use $m_{\ell \ell}^{\min }=\min \left(M_{D} / 3,2 \mathrm{TeV}\right)$.

\begin{tabular}{lrrrrrr}
\hline \hline$\sigma[\mathrm{fb}]$ & & $n=3$ & & & $n=6$ \\
$M_{D}$ & $2 \mathrm{TeV}$ & $5 \mathrm{TeV}$ & $8 \mathrm{TeV}$ & $2 \mathrm{TeV}$ & $5 \mathrm{TeV}$ & $8 \mathrm{TeV}$ \\
\hline (a) & 2270 & 1.41 & 0.0317 & 2220 & 1.36 & 0.031 \\
(b) & 408 & 1.24 & 0.0317 & 398 & 1.21 & 0.031 \\
(c) & 173 & 0.72 & 0.0204 & 66 & 0.28 & 0.008 \\
\hline \hline
\end{tabular}

interfere. For small $E_{\text {parton }}$ the interference term is significant, whereas for large $E_{\text {parton }}$ there is hardly any SM contribution.

In Table I, we show the LHC production cross section for dileptons, $n=3$ and 6 . Our fixed point results are given in (a) and (b). In (a), we retain the leading term of (7) in $s / M_{D}^{2}$. In (b), we additionally implement the leading large- $s$ suppression starting at $M_{D}$. In (c) we introduce a cutoff in $m$ and partonic energy at $M_{D}$, following [18]. For large $M_{D} \approx 5-8 \mathrm{TeV}$ the LHC has little sensitivity to quantum-gravitational effects and we find only small differences between (a) and (b). Here, the difference between (b) and (c) is due to the Kaluza-Klein sector. For small $M_{D} \approx 2 \mathrm{TeV}$, (a)-(c) lead to significant differences which originate from physics at and beyond the fundamental Planck scale, omitted in (c).

Finally, we comment on possible extensions. We have taken only the nontrivial anomalous dimension of the graviton into account, which is the dominant effect in asymptotically safe gravity. Vertex corrections can equally be studied [9]. Systematic expansions of Wilson's flow (4) in $N$-point functions work very well in other theories, e.g., infrared QCD [20]. For the physical observables studied here, we expect vertex corrections to be subleading because the relevant momentum integrals are dynamically suppressed above the Planck scale. Our simple matching in the crossover regime can be extended by implementing the explicit RG running of $\eta$, using the results of $[10,13]$. The variation in the matching scale (Fig. 2) should cover these uncertainties.

Conclusions. - We have laid out a framework to study quantum-gravitational effects at high energies within Wilson's renormalization group. We applied this to lepton pair production through virtual graviton exchange in scenarios with large extra dimensions. The main new effects are dictated by a gravitational UV fixed point above the Planck scale. Remarkably, the renormalization group improvement advocated here results in finite cross sections for gravitational lepton pair production, and leads to controlled experimental signatures at the LHC already at low integrated luminosity. This indicates that asymptotically safe gravity could be detectable at hadron colliders, pro- vided the fundamental scale of gravity is as low as the electroweak scale.

The work of D.F. L. is supported by EPSRC/STFC.

[1] N. Arkani-Hamed, S. Dimopoulos, and G. R. Dvali, Phys. Lett. B 429, 263 (1998); I. Antoniadis, N. Arkani-Hamed, S. Dimopoulos, and G. R. Dvali, Phys. Lett. B 436, 257 (1998).

[2] G. F. Giudice, R. Rattazzi, and J.D. Wells, Nucl. Phys. B544, 3 (1999); T. Han, J. D. Lykken, and R. J. Zhang, Phys. Rev. D 59, 105006 (1999).

[3] E. A. Mirabelli, M. Perelstein, and M.E. Peskin, Phys. Rev. Lett. 82, 2236 (1999); T. Han, D. L. Rainwater, and D. Zeppenfeld, Phys. Lett. B 463, 93 (1999); L. Vacavant and I. Hinchliffe, arXiv:hep-ex/0005033; J. Phys. G 27, 1839 (2001); for an overview, see, e.g., J. Hewett and M. Spiropulu, Annu. Rev. Nucl. Part. Sci. 52, 397 (2002).

[4] J. L. Hewett, Phys. Rev. Lett. 82, 4765 (1999); K. M. Cheung and G. Landsberg, Phys. Rev. D 62, 076003 (2000).

[5] S. Weinberg, in General Relativity: An Einstein Centenary Survey, edited by S.W. Hawking and W. Israel (Cambridge University Press, Cambridge, 1979), p. 790.

[6] M. Reuter, Phys. Rev. D 57, 971 (1998).

[7] W. Souma, Prog. Theor. Phys. 102, 181 (1999).

[8] O. Lauscher and M. Reuter, Classical Quantum Gravity 19, 483 (2002).

[9] R. Percacci and D. Perini, Phys. Rev. D 68, 044018 (2003); A. Codello and R. Percacci, Phys. Rev. Lett. 97, 221301 (2006); A. Codello, R. Percacci, and C. Rahmede, Int. J. Mod. Phys. A 23, 143 (2008).

[10] D. F. Litim, Phys. Rev. Lett. 92, 201301 (2004); AIP Conf. Proc. 841, 322 (2006).

[11] M. Niedermaier, Classical Quantum Gravity 24, R171 (2007). M. Niedermaier and M. Reuter, Living Rev. Relativity 9, 5 (2006).

[12] H.W. Hamber, arXiv:0704.2895; J. Ambjorn, J. Jurkiewicz, and R. Loll, Phys. Rev. Lett. 93, 131301 (2004).

[13] P. Fischer and D. F. Litim, Phys. Lett. B 638, 497 (2006); AIP Conf. Proc. 861, 336 (2006).

[14] C. Wetterich, Phys. Lett. B 301, 90 (1993).

[15] D. F. Litim, Phys. Lett. B 486, 92 (2000); Phys. Rev. D 64, 105007 (2001); Nucl. Phys. B631, 128 (2002).

[16] F. Freire, D. F. Litim, and J. M. Pawlowski, Phys. Lett. B 495, 256 (2000).

[17] G.F. Giudice and A. Strumia, Nucl. Phys. B663, 377 (2003).

[18] G. F. Giudice, T. Plehn, and A. Strumia, Nucl. Phys. B706, 455 (2005).

[19] J. Hewett and T. Rizzo, J. High Energy Phys. 12 (2007) 009.

[20] J. M. Pawlowski, D. F. Litim, S. Nedelko, and L. von Smekal, Phys. Rev. Lett. 93, 152002 (2004). 